\title{
Lin28 and Imp are Required for Stability of Bowl Transcripts in Hub Cells of the Drosophila Testis
}

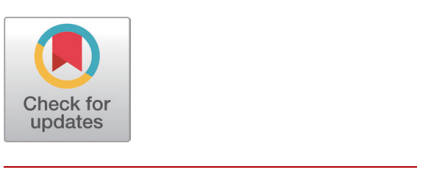

Received: October 15, 2021 Revised: November 28, 2021 Accepted: December 15, 2021

${ }^{+}$Corresponding author
Changsoo Kim
School of Biological Sciences and
Technology, Chonnam National University,
Gwangju 61186, Korea.
Tel: +82-62-530-5201
E-mail: changgk2001@hanmail.net

Copyright $\odot 2021$ The Korean Society of Developmental Biology.

This is an Open Access article distributed under the terms of the Creative Commons Attribution Non-Commercial License (http://creativecommons.org/licenses/ by-nc/4.0/) which permits unrestricted non-commercial use, distribution, and reproduction in any medium, provided the original work is properly cited.

ORCID

Van To

https://orcid.org/0000-0002-3296-4344 Hyun Ju Kim

https://orcid.org/0000-0003-4819-7431

Wijeong Jang

https://orcid.org/0000-0002-3056-4566

Perinthottathil Sreejith

https://orcid.org/0000-0001-5445-3265

Changsoo Kim

https://orcid.org/0000-0002-2852-9649

\section{Conflict of interests}

The authors declare no potential conflict of interest.

\section{Acknowledgements}

This work was supported by grants from the National Research Foundation of Korea (NRF-2021R1A2C1010334 to CK, NRF-2020R1I1A1A01074292 to WJ, NRF2017R1A6A3A11032327 to WJ). We thank the Bloomington Drosophila Stock Center (BDSC) and Vienna Drosophila RNAi

Center (VDRC) for flies, and the Drosophila Genetic Resource Center (DGRC) for Bowl cDNA. We are grateful to F. Michon for sharing Lin $28^{\mathrm{df} 30}$, T. Guy for Upd-Gal4, and

\author{
Van To, Hyun Ju Kim, Wijeong Jang, Perinthottathil Sreejith, and ${ }^{\dagger}$ Changsoo Kim \\ School of Biological Sciences and Technology, Chonnam National University, Gwangju 61186, Korea
}

\begin{abstract}
Hub cells comprise a niche for germline stem cells and cyst stem cells in the Drosophila testis. Hub cells arise from common somatic gonadal precursors in embryos, but the mechanism of their specification is still poorly understood. Here we find that RNA binding proteins Lin28 and Imp mediate transcript stability of Bowl, a known hub specification factor; Bowl transcripts were reduced in the testis of Lin28 and Imp mutants, and also when RNAmediated interference against Lin28 or Imp was expressed in hub cells. In tissue culture Luciferase assays involving the Bowl 3'UTR, stability of Luc reporter transcripts depended on the Bowl 3'UTR and required Lin28 and Imp. Our findings suggest that proper Bowl function during hub cell specification requires Lin28 and Imp in the testis hub cells.
\end{abstract}

Keywords: Lin28, Imp, Niche, Stem cells, Testis, Bowl, Drosophila

\section{INTRODUCTION}

Adult stem cells are present in most tissues and provide differentiated cells when needed throughout an animal's entire life (Kretzschmar \& Clevers, 2017). This ability is realized through a stem cell's unique capability for self-renewing cell division, in which it divides to simultaneously generate a daughter stem cell and a daughter cell that initiates differentiation (Funk et al., 2020). This unique property of stem cells is provided by the niche, a local environment that houses stem cells: a daughter cell within the niche's area of influence maintains stemness and does not undergo differentiation, while one outside the niche loses stemness and starts differentiation (Spradling et al., 2008; Losick et al., 2011). Accordingly, stem cells are attached to niche cells and influenced by signaling molecules from them.

The Drosophila testis offers an incisive genetic animal model for dissection of molecular mechanisms underlying niche specification, aging, and niche-stem cell interaction (Herrera \& Bach, 2019). An assembly of $\sim 12$ cells known as hub cells is located at the terminal tip of the testis and provides the niche for both germline stem cells (GSCs) and cyst stem cells (CySCs) (Yamashita et al., 2005). Hub cells are directly attached to GSCs and CySCs via adherens junctions, and express and secrete signaling molecules that stimulate GSCs and CySCs to promote stemness, cell division, and their attachment to hub cells (Kiger et al., 2001; Tulina \& Matunis, 2001; Shivdasani \& Ingham, 2003; Kawase et al., 2004; Leatherman \& DiNardo, 2010; Michel et al., 2011).

Within the Drosophila testis, the RNA binding protein Lin28 is exclusively expressed in hub cells (Sreejith et al., 2019). Lin28 not only acts as a repressor of Let-7 biogenesis but also controls the 
N Sokol for Lin2 $28^{\Delta 1}$.

Authors' contributions

Conceptualization: $\operatorname{Kim} \mathrm{C}$.

Data curation: To V, Kim HJ.

Methodology: Jang W, Sreejith P.

Writing-original draft:To V, Kim H

Writing-review \& editing: Kim C.

\section{Ethics approval}

This article does not require IRB/IACUC approval because there are no human and animal participants. stability and translation of target mRNAs including Unpaired (Upd), an extrinsic stem cell selfrenewal factor that is expressed and secreted from hub cells (Kiger et al., 2001; Tulina \& Matunis, 2001; Sreejith et al., 2019). A second RNA binding protein from a conserved protein family, IGFII messenger RNA binding protein (Imp), is also required for stabilizing Upd transcripts (Toledano et al., 2012). However, what other factors Lin28 and Imp may act to stabilize in hub cells is yet largely unknown.

Hub cells and CySCs are both specified during embryogenesis from somatic gonadal precursors (SGPs) (D Le Bras \& Van Doren, 2006; Kitadate \& Kobayashi, 2010; iNardo et al., 2011; Okegbe $\&$ DiNardo, 2011). For hub cells, specification, aggregation, and assembly are known to require Notch signaling and the transcription factor Bowl, while CySC specification requires Lines (DiNardo et al., 2011; Okegbe \& DiNardo, 2011). Notably, the embryonic gonads of Bowl mutants contain fewer hub cells, while increased Bowl activity transforms CySCs to the hub cell fate (DiNardo et al., 2011); these findings indicate that Bowl is a hub cell specification factor. Similarly, Lin28 mutant embryonic gonads contain fewer hub cells (Sreejith et al., 2019), suggesting that Lin28 could function in hub cell specification together with Bowl. Here we find that in hub cells, Bowl transcripts require Lin28 and Imp for stability.

\section{RESULTS AND DISCUSSION}

Lin28 was previously shown to be required for stabilizing transcripts of Upd, an important hub cell factor (Sreejith et al., 2019). This observation prompted us to identify other targets that are stabilized by Lin28 in hub cells of the Drosophila testis. To identify new target transcripts, we carried out reverse transcription polymerase chain reaction (RT-PCR) and compared transcript levels in Lin28 mutant and wild-type testis. This screening identified Bowl transcripts as reduced in the Lin28 mutant (Fig. 1). Lin28 is exclusively expressed in hub cells, suggesting that Bowl reduction is due to Lin28 mutation specifically in hub cells. To confirm this notion with an independent method, we used the bipartite GAL4-UAS system (Brand et al., 1994) in combination with RNA-mediated interference (RNAi) to reduce Lin28 function exclusively in hub cells. Transgenic flies bearing Upd-Gal4 (hub cell-specific Gal4) and UAS-Lin28 RNAi were generated (Upd-Gal4>UAS-Lin28 RNAi), in which Lin28 RNAi was specifically expressed in hub cells. RT-PCR of testis samples from two independent Lin28 RNAi lines confirmed the reduction of Bowl transcripts (Fig. 2). We likewise examined the relation of Bowl and Imp; however, Imp null mutation was lethal, so we instead relied on Imp knockdown in hub cells of the adult testis. Testis from three independent lines of Upd-Gal4>UAS-Imp RNAi flies expressing Imp RNAi in hub cells likewise showed reduction of Bowl transcripts (Fig. 2). Taken together, these data suggest that maintenance of Bowl transcripts in hub cells requires both Lin28 and Imp.

To further investigate the molecular mechanisms underlying promotion of Bowl transcript stability by Lin28 and Imp, we carried out cell culture experiments using Luciferase (Luc) constructs bearing the Bowl 3'UTR (Luc-Bowl 3'UTR), employing RT-PCR to examine whether those hybrid transcripts were stabilized by Lin28 and Imp, which stabilizes target mRNAs through 3'UTR (Toledano et al., 2012; Lee et al., 2019; Sreejith et al., 2019). When either Lin28 or Imp was co-transfected with the construct, Luc-Bowl 3'UTR transcript levels were increased (Fig. 3A). Meanwhile, levels of control Luc transcripts lacking the Bowl 3'UTR were not affected by the presence of either Lin 28 or Imp (Fig. 3B). These results suggest that Lin28 and Imp stabilize LucBowl 3'UTR transcripts through the Bowl 3'UTR.

Fig. 4 illustrates our proposed model of the roles of Lin28 and Imp in the testis. We and others 
A

Lin28 Wild-type
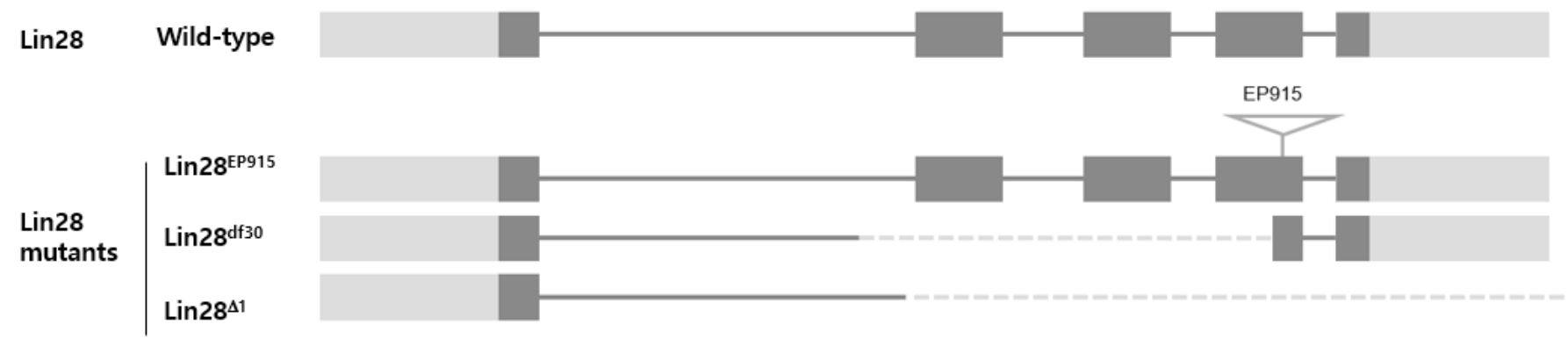

B

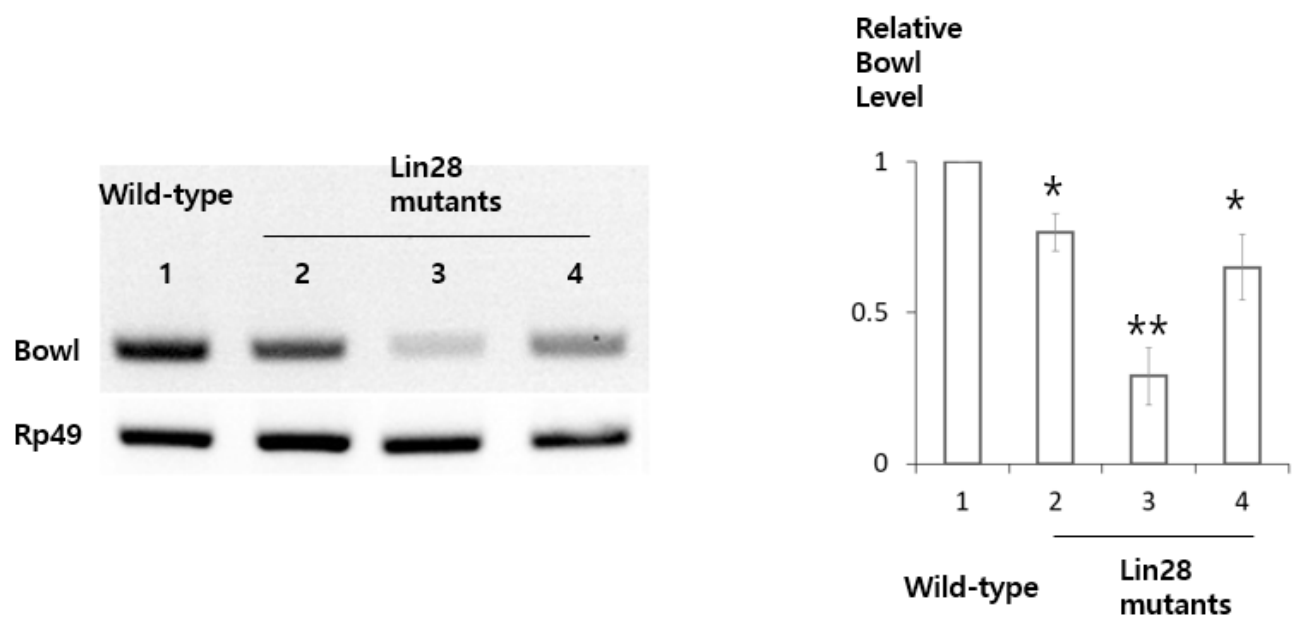

Fig. 1. Reduced Bowl transcripts in Lin28 mutant testis. (A) Lin28 mutants with a P-element insertion in the $4^{\text {th }}$ exon (Lin28 $8^{\text {EP915 }}$ ) and with deletions in the middle of an exon as indicated (Lin2 $8^{\mathrm{d} 300}$ and Lin28 ${ }^{\Delta 1}$ ). (B) (Left) Agarose gel image of RT-PCR products from testis with Lin28 mutations. Rp49 used as a loading control. 1. Wild type, 2. Lin28 ${ }^{\mathrm{EP} 915}, 3$. Lin28 ${ }^{\mathrm{d} 330}, 4$. Lin28 ${ }^{\Delta 1}$. (Right) Quantification of RT-PCR bands by Image J analysis, with Bowl band intensity normalized by control Rp49 band intensity. 1. Wild type, 2. Lin28 $8^{\mathrm{EP} 915}, 3$. Lin28 ${ }^{\mathrm{d} 30}, 4$. Lin28 $8^{\Lambda 1}$. $p$-values by student's $t$-test in Sigma-Aldrich Plot. * $p<0.05,{ }^{* *} p<0.01$. RT-PCR, reverse transcription polymerase chain reaction.

\section{MATERIALS AND METHODS}

\section{Drosophila strains}

Flies were grown in standard fly food. $\mathrm{W}^{1118}$, Lin28 ${ }^{\mathrm{EP} 915}$, and Imp ${ }^{\mathrm{MI} 05901}$ were from the 
$\begin{array}{lll}\text { Upd-Gal4 } & \text { Upd-Gal4 } & \text { Upd-Gal4 } \\ & \text { Imp RNAi } & \text { Lin28 RNAi }\end{array}$
1
2
3
4
5
6

Bowl

Rp49

\section{Relative \\ Bowl \\ Level}

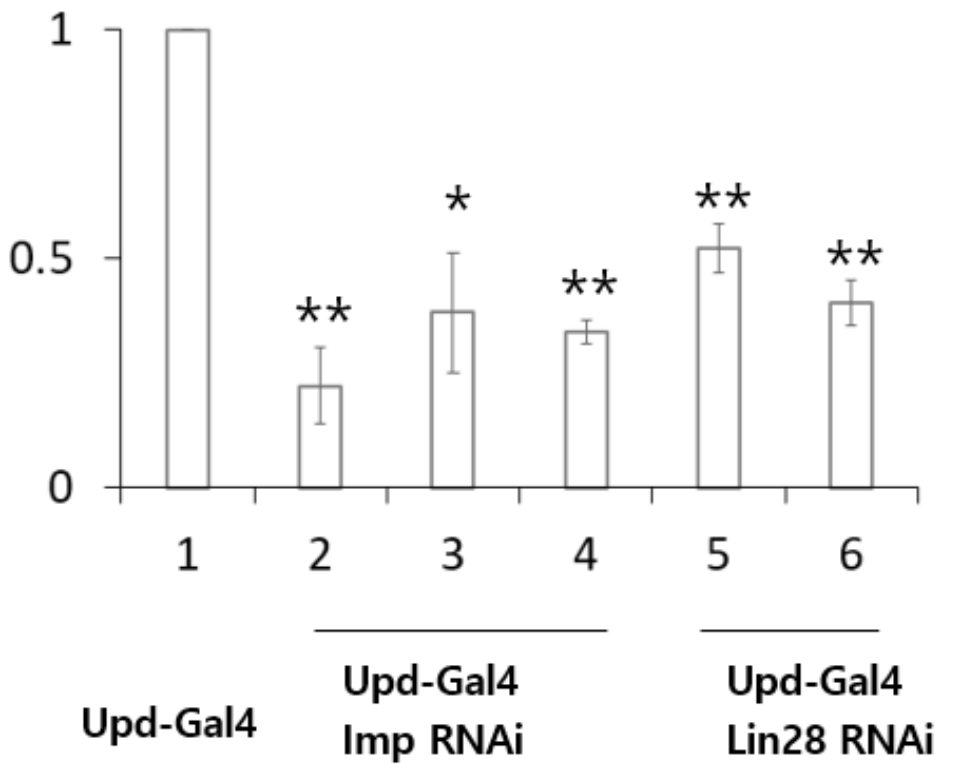

Fig. 2. Reduced Bowl transcripts in the testis with Lin28 and Imp RNAi expressed in the hub cells. (Up) Agarose gel image of RT-PCR products from testis with RNAi expressed in hub cells. Rp49 used as a loading control. 1. Upd-Gal4, 2-4. Upd-Gal4>UAS-Imp RNAi (2032, 20322, 34977), 5-6. UpdGal4>UAS-Lin28 RNAi (50679, 29564). (Down) Quantification of RT-PCR bands by Image J analysis, with Bowl band intensity normalized by control Rp49 band intensity. 1. Upd-Gal4, 2-4. Upd-Gal4>UASImp RNAi (2032, 20322, 34977), 5-6. Upd-Gal4>UAS-Lin28 RNAi $(50679,29564)$. p-values by student's $t$-test in Sigma-Aldrich Plot. * $p<0.05,{ }^{* *} p<0.01$. RT-PCR, reverse transcription polymerase chain reaction; RNAi, RNA-mediated interference; Upd, unpaired.

Bloomington Drosophila Stock Center (BDSC). Lin2 ${ }^{\mathrm{d} 330}$ was from F. Michon and Lin2 $8^{\Delta 1}$ from N. Sokol. UAS-Lin28 RNAi $(50679,29564)$ and UAS-Imp RNAi (34977) were from the BDSC and UAS-Imp RNAi $(20321,20322)$ from the Vienna Drosophila RNAi Center (VDRC). 
A

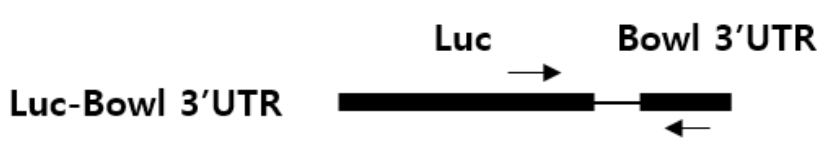

Luc-Bowl 3'UTR
B

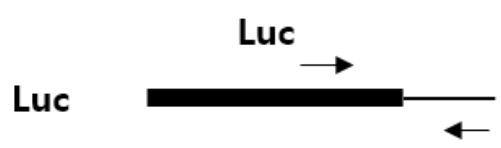

Luc-Bowl 3'UTR

Rp49

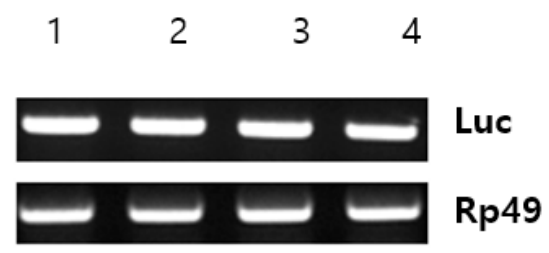

Luc

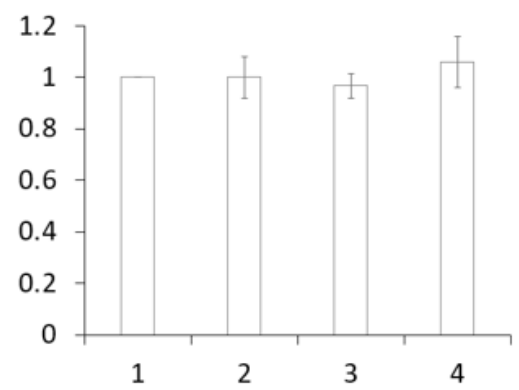

Fig. 3. Bowl 3'UTR mediates stability of Bowl transcripts. Agarose gel image of RT-PCR products from S2 cells co-transfected with the Luc-Bowl 3'UTR hybrid construct (A) and parental Luc construct (B) in combination with parental vector (1), Lin28 expression vector (2), Imp expression vector (3) and Lin28+Imp (4). Rp49 used as a loading control. Primers used for RT-PCR indicated as arrows. (Bottom) Quantification of RT-PCR bands by Image J analysis, with Bowl band intensity normalized by control Rp49 band intensity. $p$-values by student's $t$-test in Sigma-Aldrich Plot. ${ }^{*} p<0.05$, ${ }^{* *} p<0.01$. RT$\mathrm{PCR}$, reverse transcription polymerase chain reaction.

\section{Reverse transcription polymerase chain reaction (RT-PCR)}

RNA was extracted using standard TRIzol (Invitrogen) procedure. Complementary DNA synthesis was performed with oligodT primers using one microgram RNA. PCR was performed with the following primers: Rp49: Forward 5'-CACCAGGAACTTCTTGAATCCGG-3', Reverse 5'-AGATCGTGAAGAAG CGCACC-3'; Bowl: Forward 5'-CTGCTCATCCACGAGAGG-3', Reverse 5'-TGTGGACAGCCAAGGTTC-3'; Luc-Bowl 3'UTR: Forward 5'-CC CTCGAG CAATCCATTAATGGAG-3', Reverse 5'-GC TCTAGA CATTATTAATGCATACTTTATTTGA-3'. RT-PCR were carried out three replicates in Fig. 1, 2 , and 3 .

\section{Cloning of Luc-Bowl 3'UTR}

pAc5.1A-Luc (S2 cell expression vector) was created using the Luc gene from the pGL3-Basic vector (Promega, Madison, WI, USA), which was ligated into the EcoRI-NotI sites of pAc5.1/V5HisA (Invitrogen). The Bowl 3'UTR (853 bp) from Bowl cDNA (RE05342, Drosophila Genetic Resource Centre) was ligated into the XhoI-XbaI sites of the pAc5.1A-Luc vector. 
A

Adult gonad

(testis)

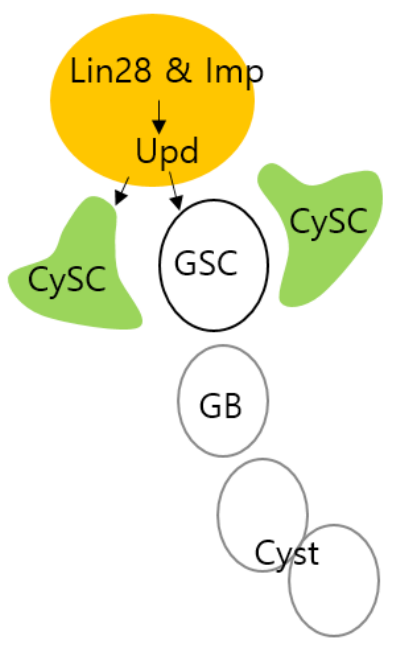

B

$$
\text { Embryo gonad }
$$

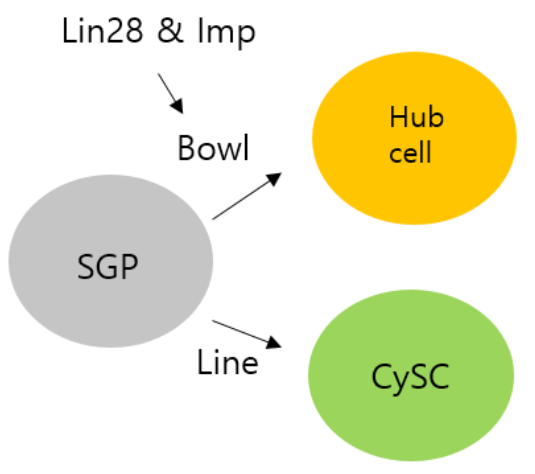

Fig. 4. Models depicting roles of Lin28 and Imp in the Drosophila testis. (A) Roles of Lin28 and Imp in hub cells of the adult testis. Upd transcripts are stabilized by Lin28 and Imp. GSC, germline stem cell; CySC, cyst stem cell; GB, goniablast. (B) Roles of Lin28 and Imp in hub cell specification in the embryonic gonads. Bowl transcripts are stabilized by Lin28 and Imp. SGP, somatic gonadal precursor. Upd, unpaired.

\section{S2 cell culture and transfection}

Schneider 2 cells were grown and maintained in Shields and Sang M3 insect Media (SigmaAldrich, St. Louis, MO, USA) supplemented with $10 \%$ insect media supplement (Sigma-Aldrich) with appropriate antibiotics at $25^{\circ} \mathrm{C}$. Cells were subcultured every five days at a 1:5 ratio when confluency reached around $100 \%$. The DDAB method of transfection was employed with some modifications for 24-well plates (Han, 1996). Cells were collected and assayed three days after transfection.

\section{REFERENCES}

Brand AH, Manoukian AS, Perrimon N (1994) Ectopic expression in Drosophila. Methods Cell Biol 44:635-654.

DiNardo S, Okegbe T, Wingert L, Freilich S, Terry N (2011) Lines and bowl affect the specification of cyst stem cells and niche cells in the Drosophila testis. Development 138:16871696.

Funk MC, Zhou J, Boutros M (2020) Ageing, metabolism and the intestine. EMBO Rep 21:e50047.

Han K (1996) An efficient DDAB-mediated transfection of Drosopbila S2 cells. Nucleic Acids Res 24:4362-4363.

Herrera SC, Bach EA (2019) JAK/STAT signaling in stem cells and regeneration: From Drosophila to vertebrates. Development 146:dev167643.

Kawase E, Wong MD, Ding BC, Xie T (2004) Gbb/Bmp signaling is essential for maintaining germline stem cells and for repressing bam transcription in the Drosopbila testis. Development 


\section{1:1365-1375}

Kiger AA, Jones DL, Schulz C, Rogers MB, Fuller MT (2001) Stem cell self-renewal specified by JAK-STAT activation in response to a support cell cue. Science 294:2542-2545.

Kitadate Y, Kobayashi S (2010) Notch and Egfr signaling act antagonistically to regulate germline stem cell niche formation in Drosophila male embryonic gonads. Proc Natl Acad Sci USA 107:14241-14246.

Kretzschmar K, Clevers H (2017) Wnt/ $\beta$-catenin signaling in adult mammalian epithelial stem cells. Dev Biol 428:273-282.

Le Bras S, Van Doren M (2006) Development of the male germline stem cell niche in Drosophila. Dev Biol 294, 92-103.

Leatherman JL, DiNardo S (2010) Germline self-renewal requires cyst stem cells and stat regulates niche adhesion in Drosophila testes. Nat Cell Biol 12:806-811.

Lee M, Nguyen TMT, Kim K (2019) In-depth study of lin-28 suggests selectively conserved let-7 independent mechanism in Drosophila. Gene 687:64-72.

Losick VP, Morris LX, Fox DT, Spradling A (2011) Drosopbila stem cell niches: A decade of discovery suggests a unified view of stem cell regulation. Dev Cell 21:159-171.

Michel M, Raabe I, Kupinski AP, Pérez-Palencia R, Bökel C (2011) Local BMP receptor activation at adherens junctions in the Drosophila germline stem cell niche. Nat Commun 2:415.

Okegbe TC, DiNardo S (2011) The endoderm specifies the mesodermal niche for the germline in Drosophila via Delta-Notch signaling. Development 138:1259-1267.

Shivdasani AA, Ingham PW (2003) Regulation of stem cell maintenance and transit amplifying cell proliferation by tgf- $\beta$ signaling in Drosophila spermatogenesis. Curr Biol 13:2065-2072.

Spradling AC, Nystul T, Lighthouse D, Morris L, Fox D, Cox R, Tootle T, Frederick R, Skora A (2008) Stem cells and their niches: Integrated units that maintain Drosopbila tissues. Cold Spring Harb Symp Quant Biol 73:49-57.

Sreejith P,Jang W, To V, Hun Jo Y, Biteau B, Kim C (2019) Lin28 is a critical factor in the function and aging of Drosophila testis stem cell niche. Aging 11:855-873.

Toledano H, D'Alterio C, Czech B, Levine E, Jones DL (2012) The let 7-Imp axis regulates ageing of the Drosophila testis stem-cell niche. Nature 485:605-610.

Tulina N, Matunis E (2001) Control of stem cell self-renewal in Drosophila spermatogenesis by JAK-STAT signaling. Science 294:2546-2549.

Yamashita YM, Fuller MT, Jones DL (2005) Signaling in stem cell niches: Lessons from the Drosophila germline. J Cell Sci 118:665-672. 
